УДК 532.5

\title{
The Study of Ethanol and Water Mixing Modes in the T-shaped Micromixers
}

\author{
Alexander S. Lobasov* \\ Anna A. Shebeleva ${ }^{\dagger}$ \\ Andrey V. Minakov $\ddagger$ \\ Institute of engineering physics and radio electronics \\ Siberian Federal University \\ Kirensky, 28, Krasnoyarsk, 660074 \\ Institute of Thermophysics SB RAS \\ Lavrent'ev, 1, Novosibirsk, 630090 \\ Russia
}

Received 04.07.2018, received in revised form 10.09.2018, accepted 28.11.2018

The study of regimes of ethanol and water mixing was carried out using numerical simulation, and the dependence of the mixing efficiency of these fluids on the Reynolds number was defined. In the course of the numerical investigation, a significant effect of the differences in the viscosities and densities of the miscible fluids on the flow regimes and the mixing efficiency of these two fluids was found. The distributions of pressure, components concentration and velocity in the micromixer were obtained. The dependence of the fluids mixing efficiency and pressure drop on the Reynolds number, as well as the map of flow and mixing regimes were determined. The nonlinear dependence of the mixture thermophysical properties on the ethanol concentration was taken into account. It was found that the last one results in much higher pressure drop comparing to the case of the water-water mixing. Moreover, an inflection was observed in the dependence of the pressure drop on the Reynolds number for the water-ethanol mixing at the transition of the flow in a micromixer to the engulfment regime.

Keywords: hydrodynamics, numerical simulation, T-shaped micromixer, micromixer, ethanol, nonlinear. DOI: $10.17516 / 1997-1397-2019-12-2-202-212$.

\section{Introduction}

The microchannel devices are widely used in various fields of science and technology as microreactors, microscale heat exchangers, micromixers, etc. The micromechanics has become a rapidly developing and promising research area in recent years due to the miniaturization of technological processes in the chemical industry. Many studies have noted that the use of microdevices allows significantly enhancing the physicochemical processes in comparison to classical space consuming reactors [1-3]. However, despite the obvious benefits of microchannel technology, there are a number of specific problems. Most microchannel devices that used in chemistry and biology require fast and effective mixing of substances [1-5]. At the same time, the flow in microchannels is predominantly laminar and mixing occurs through diffusion, and thus very

\footnotetext{
*perpetuityrs@mail.ru

†ashebeleva@sfu-kras.ru

‡aminakov@sfu-kras.ru

(c) Siberian Federal University. All rights reserved
} 
slowly. Therefore, the design and optimization of micromixers with the shortest mixing time is an actual problem in the development of microchannel devices. The T-shaped micromixers (Fig. 1) are the simplest in the manufacture in terms of their underlying geometry. There are many experimental and numerical investigations of flow and mixing regimes in such micromixers [6-26]. Five different flow regimes were revealed in the researches of mixing processes of two fluids with the same thermophysical properties. Furthermore, a dramatic increase in mixing efficiency at the transition from the symmetric stationary flow regime to the asymmetric stationary one (otherwise called the engulfment regime) was noted in most of research works. That regime is of the greatest interest for researchers because the mixing efficiency increases abruptly in this mode while the pressure drop in the mixing channel increases insignificantly. On the other side, the investigations of mixing of two fluids with different thermophysical properties were carried out by many researchers [14-20]. For example, a systematic study of the viscosity effect on mixing modes in the T-shaped micromixer was carried out in [14], where the significant effect of viscosities ratio on the flow structure before and after the transition from symmetric to asymmetric flow regimes was shown. The self-similar behaviour of the asymmetric flow regime was observed. A systematic study of the density effect on mixing modes in the T-shaped micromixer was carried out in [15], and it was shown that the change in the density of one of the miscible fluids does not lead to a shift of flow regimes. However, a significant flow asymmetry in the region before its transition to the engulfment flow mode was observed. As a rule, the problem set up for a numer-

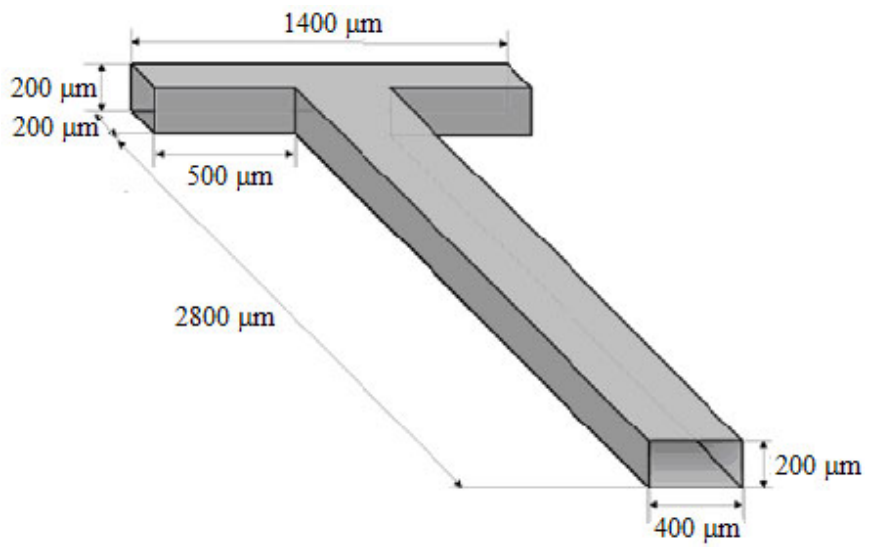

Fig. 1. Geometric configuration of the task

ical study considers the "model" fluids with specified properties that do not exist in nature. The amount of studies of mixing really existing fluids is small. For example, water-ethanol mixing in T-shaped microdevices was investigated numerically in [19] and experimentally in [20] taking into account both density and viscosity variations. The results were compared with studies of water-water mixing. In the both research works, it was shown that at smaller Reynolds number there is no vortices formation for either cases, and at larger Reynolds numbers, mixing of water and ethanol may take considerably longer, as the onset of engulfment is retarded and occurs at larger Reynolds number. The critical Reynolds number at which the engulfment flow regime occurs differs for different mixtures, also it strongly depends on the channel geometry, and e.g., for water-water mixing in the T-channel, shown in Fig. 1 the critical Reynolds number is equal to 145 . The aim of the present work is to carry out systematic investigation of the water-ethanol mixing in the T-shaped micromixer, to compare the obtained results with the results of studies of 
water-water mixing, to determine the critical Reynolds number, and to investigate the influence of non-linear dependence of mixture properties on the flow and mixing regimes.

\section{Mathematical Model and Numerical Algorithm}

Incompressible flows of multi-component and generally non-Newtonian fluids, which are described using a hydrodynamic approach based on the solution of the Navier-Stokes equations were considered in this work. Currently, in numerous experiments it was shown that such description for fluids works well for the channels sizes up to $\mu \mathrm{m}$.

In general, the Navier-Stokes equations system has the following form:

$$
\frac{\partial \rho}{\partial t}+\nabla(\rho v)=0, \quad \frac{\partial \rho v}{\partial t}+\nabla(\rho v v)=-\nabla p+\nabla T .
$$

Here $\mathbf{r}$ is the fluid density, $p$ is the pressure, $\mathbf{v}$ is the velocity, and $\mathbf{T}$ is the viscous stress tensor.

The effective viscosity and the density of the mixture are determined through the mass fraction of the first component $f$ and effective viscosities $\mathrm{m}_{1,2}$ and partial densities $\mathrm{r}_{1,2}$ of pure components, respectively:

$$
\mu=f \mu_{1}+(1-f) \mu_{2}, \quad \frac{1}{\rho}=\frac{f}{\rho_{1}}+\frac{(1-f)}{\rho_{2}} .
$$

Here the evolution of mass concentrations is determined by the equation:

$$
\frac{\partial \rho f}{\partial t}+\nabla(\rho f v)=\nabla(\rho D \nabla f)
$$

where $D$ is the diffusion coefficient.

A software package for computational fluid dynamics was used to solve the equations system described above. The detailed description of the numerical algorithm of this software tool is given in [27]. Here the main points of numerical technique will be noted. The difference analogue of the convective-diffusion equations (1) and (2) is derived using the finite volume method [28-30] for structured multi-block grids. In this case, the resulting scheme is automatically conservative. The method consists in splitting the computational domain into control volumes and integrating the original conservation equations for each control volume to obtain finite-difference equations. Approximation of convective terms of equations (1) and (2) is carried out using the second order upwind QUICK [31] and TVD [30] schemes, respectively. To approximate the unsteady terms of hydrodynamics equations a second order implicit scheme was applied. Diffusion fluxes and source terms were approximated by finite-volume analogues of the central-difference schemes with the second order of accuracy. The coupling between the velocity and pressure fields, ensuring the fulfillment of the continuity equation, was implemented by means of the SIMPLEC procedure for combined grids [28]. Rhi-Chow approach, consisting in the introduction of monotonizator into the equation for the pressure correction [32] was used to eliminate the pressure field oscillations. The obtained difference equations resulting from discretization of the original system of differential equations were solved by the iterative method using algebraic multigrid solver [33].

Three-block grid consisting of 9.5 million cells was used for calculations in this work. Preliminary calculations have shown that this level of grid resolution is acceptable in terms of calculation accuracy. A constant fluid flow rate with steady-state velocity profile was set at the inlets of micromixer. The Neumann conditions, meaning zero derivatives of all scalar quantities along 
the normal to the boundary were set at the outlet of the mixing channel. For the velocity vector components, no-slip condition was taken as a boundary condition on the channels walls. The applicability of this type of boundary conditions for channels sizes up to $50 \mu \mathrm{m}$ has been demonstrated in $[1,12,13]$.

The channel dimensions are shown in Fig. 1. The channel thickness is $200 \mu \mathrm{m}$, while the width of its narrow and wide parts is $200 \mu \mathrm{m}$ and $400 \mu \mathrm{m}$, respectively.

The pressure drop in the mixing channel, as well as the mixing efficiency were determined in this study. The mixing efficiency is usually quantified using the parameter $M=1-\left(\sigma / \sigma_{0}\right)^{0.5}$, where $\sigma=V^{-1} \int_{V}(f-\langle f\rangle)^{2} d V$ is the root-mean-square deviation of the mass fraction of mixture component $f$ from its average value $\langle f\rangle, \sigma_{0}=\langle f\rangle \cdot(1-\langle f\rangle)$ is the maximum root-mean-square deviation, and $V$ is the volume of the computational domain.

\section{Numerical simulation results}

There are many experimental and numerical investigations of flow and mixing regimes of two fluids with the same thermophysical properties in T-shaped micromixers [6-13,21-26]. Five different flow regimes were revealed in the researches of mixing processes: stationary vortex-free flow; stationary symmetric vortex flow; stationary asymmetric vortex flow (engulfment regime); unsteady periodic flow; stochastic flow, laminar-turbulent transition. Furthermore, a dramatic increase in mixing efficiency at transition from symmetric to asymmetric mode for stationary flow regime (called the engulfment regime) was noted in most of research works. That regime is of the greatest interest for researchers because the mixing efficiency increases abruptly in this mode while the pressure drop in the mixing channel increases insignificantly. The same flow regimes with some differences can be found for water-ethanol mixing.

The numerical investigation of flow and mixing regimes of fluids with different thermophysical properties was carried out. Pure water was supplied to mixer through one of the inlets with mass flow rate $Q_{i n}$. Ethyl alcohol was supplied through another inlet with the same mass flow rate. As is known, the dependence of the density and, especially, the viscosity of the waterethanol mixture on the concentration of the ethyl alcohol are significantly nonlinear. Therefore, investigation of the mixing of these fluids using a nonlinear dependence of the thermophysical properties of the solution on the concentration was carried out. These dependencies were experimentally obtained in [34] and are shown in Fig. 2. As can be seen, the maximum value of the mixture viscosity is observed at $40 \%$ alcohol concentration, and it is 2.9 times higher than the water viscosity. The viscosity of ethyl alcohol is 1.2 times higher, and the density is 1.27 times lower than the corresponding values of water. The diffusion coefficient of ethanol in water is $D=1.24 \times 10^{-9} \mathrm{~m}^{2} / \mathrm{s}$.

The change in the flow regimes in the microchannel is characterized by Reynolds number, determined as $\operatorname{Re}=\rho U d_{h} / \mu$, where $U=Q /\left(2 \rho H^{2}\right)=Q_{i n} /\left(\rho H^{2}\right)$ is the superficial velocity in the mixing channel, $H=200 \mu \mathrm{m}$ is the channel height, and $d_{h}=267 \mu \mathrm{m}$ is the hydraulic diameter. For very small values of Reynolds numbers $(\mathrm{Re}<10)$ the flow and mixing structure of fluids with different properties in general is similar to the flow of two identical fluids. The Reynolds number equal to 1 corresponds to creeping stratified flow accompanied by a weak mixing. The concentration contours for different Reynolds numbers are shown in Fig. 3. Here and below the red color corresponds to $100 \%$ concentration of water, and the blue color corresponds to $100 \%$ concentration of ethanol. As can be seen from this figure, due to higher viscosity of the ethanol, 

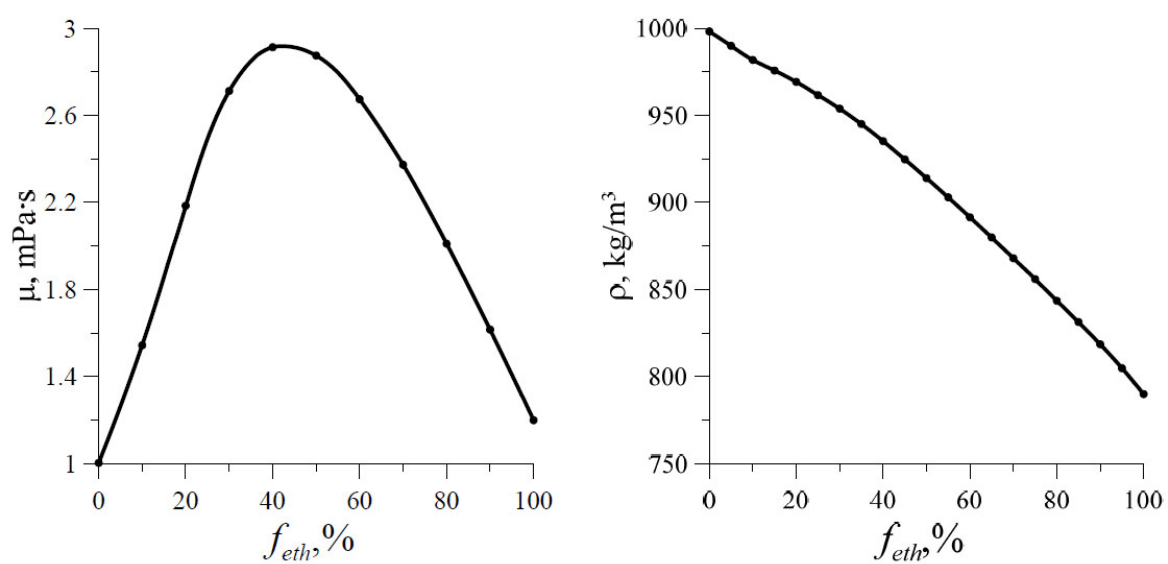

Fig. 2. Dependence of viscosity and density of water-ethanol mixture on the mass concentration of the ethyl alcohol

the water is pushed by the last one to the channel wall. The velocity profiles in the central section of the channel are shown in Fig. 4. As one can see from that figure, the water velocity is lower than the ethanol velocity due to the higher density of the former.

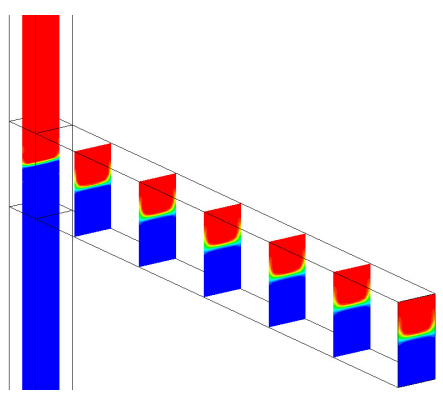

a)

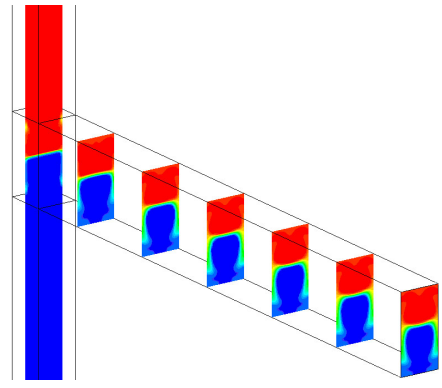

b)

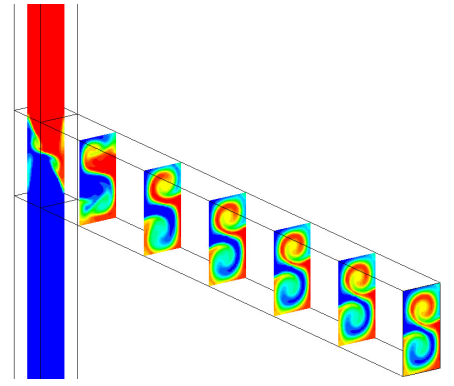

c)

Fig. 3. Concentration isolines of water-ethanol mixture, Re: 50 (a); 120 (b); 200 (c)

Further, from the Re $\sim 120$ (see Fig. 4), a pair of symmetrical horseshoe vortices begin to develop near the left end wall of the mixer and propagate into the mixing channel. In this case, due to the difference in viscosities, an asymmetry of the vortices is manifested, at that for a more viscous fluid (ethyl alcohol) that vortex is in a rudimentary stage, whereas for water it is clearly visible. At $\operatorname{Re} \sim 140$, the vortex in ethanol is already noticeable, but it is still substantially smaller than the vortex for water. With an increase in the Reynolds number, both of these vortices become more pronounced, but a significant difference in the dimensions of the vortices remains. The ethyl alcohol gradually starts to "flow around" the water, as it was shown for the case of different viscosities [14] (see Fig. 3). With a further increase in the Reynolds number, the given structure develops, the vortices grow and the effect of flowing of the one fluid around another intensifies. On the other hand, the density of the ethyl alcohol is 1.27 times lower than that of the water and the latter in turn begins to "flow around" the former. The subsequent increase in the Reynolds number leads to a gradual development of these two effects, while the flow remains symmetrical with respect to the horizontal plane.

It was shown earlier [14] that the onset of the engulfment regime depends only on the viscosi- 


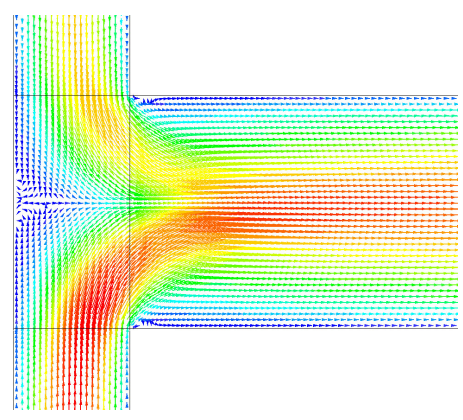

a)

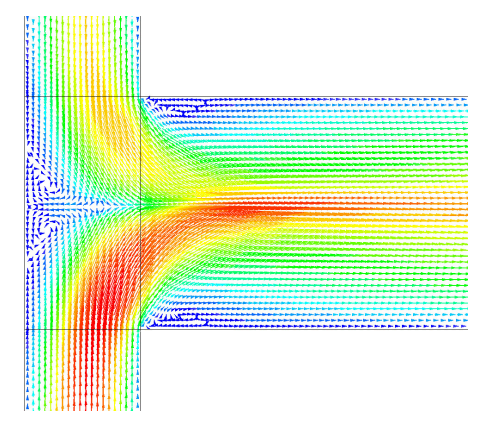

b)

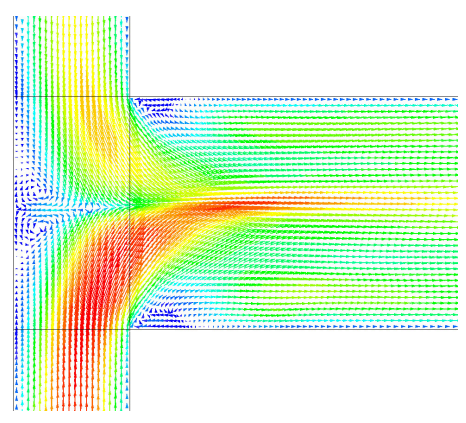

c)

Fig. 4. Velocity profiles in the central cross-section, Re: 50 (a); 120 (b); 200 (c)

ties ratio and does not depend on the densities ratio. Therefore, using the viscosity coefficient $C=2 \mu_{0}\left(\mu+\mu_{0}\right)^{-1}$, it can be assumed that this transition will occur at $\operatorname{Re} \sim 162$ and indeed, the transition occurs in this area. Further, with an increase in the Reynolds number, this type of flow develops, an increase in the vortical motion intensity, in the interface area of the media, and, accordingly, in the mixing efficiency occurs. From the Reynolds number of about 225, the transition to the next regime, where the flow ceases to be steady and becomes periodic, starts. To understand the reason why it happens, it is necessary to consider the region immediately near the inlet of the mixing channel. It turns out that, even at $\operatorname{Re} \sim 10$, the shown-above double vortex forms in the water flow just behind the entrance to the mixing channel. At such a small Reynolds number, these vortices almost immediately dissipate, and with a further increase in the Reynolds number they move deeper into the channel, dissipating at a farther distance from the entrance to the mixing channel.

The vortices structure at different Reynolds numbers is shown in Fig. 5 through the isosurfaces of $\lambda_{2}$ that is the second eigenvalue of the tensor $\left(\mathbf{S}^{2}+\Omega^{2}\right)$, where $\mathbf{S}$ is the strain rate tensor and $\Omega$ is the vorticity tensor. In addition, it can be seen from that figure that a similar two-vortex structure also appears and continues to develop in the ethanol stream, but at higher Reynolds numbers than for water. As already mentioned above, the transition to the engulfment regime occurs in the region of $\operatorname{Re} \sim 162$. That transition, as for all other cases, is pronounced, i.e., occurs in a very narrow range of Reynolds numbers. That can be seen from Fig. 6, which represents the isolines of the water and ethanol concentrations, as well as velocity profiles in the cross section at Reynolds numbers 156, 160-163 and 168. Further, with an increase in the Reynolds number, this type of flow develops, an increase in the vortical motion intensity occurs, the interface area of the media, and, accordingly, the mixing efficiency enhances. As can be seen from that figure, when the flow approaches the transition region, it gradually loses symmetry with respect to the horizontal plane, and as the Reynolds number becomes equal to 162, the flow rearranges. The velocity profiles in the cross section of the mixing channel outlet for the same Reynolds numbers are shown in Fig. 6 for higher clarity.

As was mentioned above, the double-vortex structure, that forms in the flow of each fluid, appears at a certain Reynolds number, but, depending on the fluid viscosity, either reaches the exit from the channel, or has time to "dissipate". A double vortex in the flow of water for such Reynolds numbers continues until the outlet of the channel. A double vortex in the ethyl alcohol exists only at a certain distance from the entrance to the mixing channel. It was found that each of the double-vortex structures lose symmetry with respect to the horizontal plane when approach the transition point, and at the moment of transition one of the vortices in each of the 


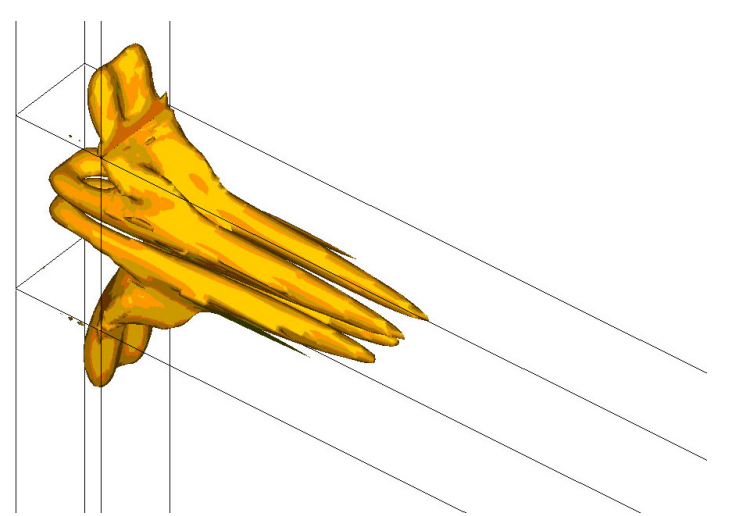

a)

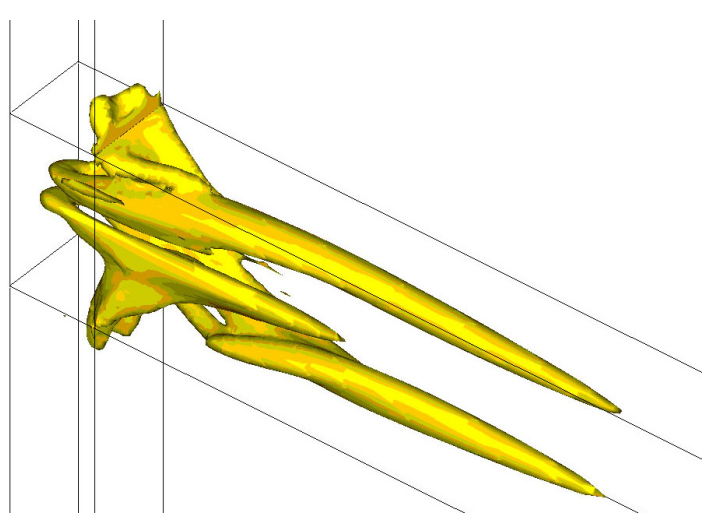

b)

Fig. 5. $\lambda_{2}$ isosurfaces, Re: 120 (a); 200 (b)

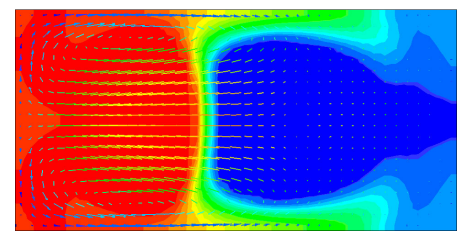

a)

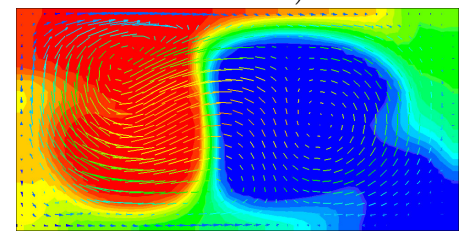

d)

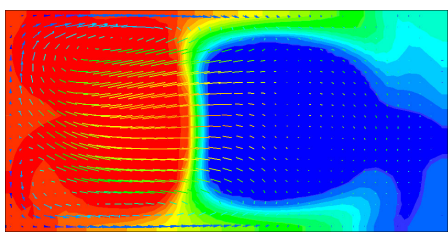

b)

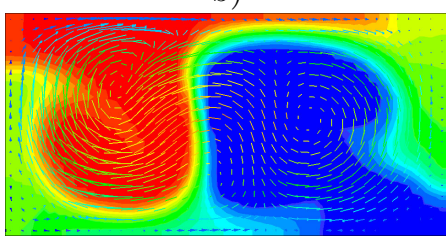

e)

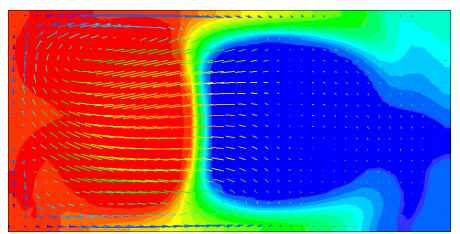

c)

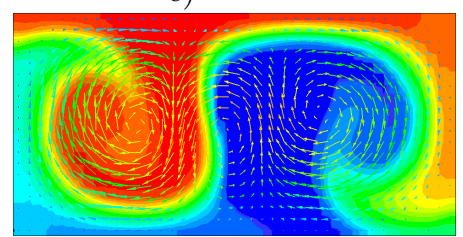

f)

Fig. 6. Velocity profiles and concentration isolines, Re: 156 (a); 160 (b); 161 (c); 162 (d); 163 (e); 168 (f)

fluids begins to predominate and the second to attenuate. As a result, two weaker vortices merge into one large vortex, and the flow structure actually converts into a three-vortex system. That structure moves into the depth of the mixing channel and continues to exist in this form until the outlet of this channel. Apparently, the sharp increase in the value of the mixing efficiency in that region explains by the appearance of the third vortex. With a subsequent increase in the Reynolds number, the engulfment regime becomes more pronounced, and the transitional three-vortex structure converts into a two-vortex structure (see Fig. 6f).

It is suitable to plot the mixing efficiency as a function of the reduced Reynolds number using the formula given for the case of different viscosities [14] $\left(\operatorname{Re}_{r d}=\operatorname{Re} \cdot C\right)$ and compare the obtained dependences with results of mixing of the fluids with the same properties. The dependence of the mixing efficiency of various fluids on the reduced Reynolds number is shown in Fig. 7a. In addition, the pressure drop between the inlet and outlet of the mixing channel of the T-micromixer as a function of the Reynolds number was plotted for these cases (see Fig. 7b). The nonlinearity of properties significantly influenced on the mixing efficiency and, especially, on the pressure drop in the engulfment regime.

Analyzing that graph, one can see that the pressure drop in the case of a nonlinear dependence 


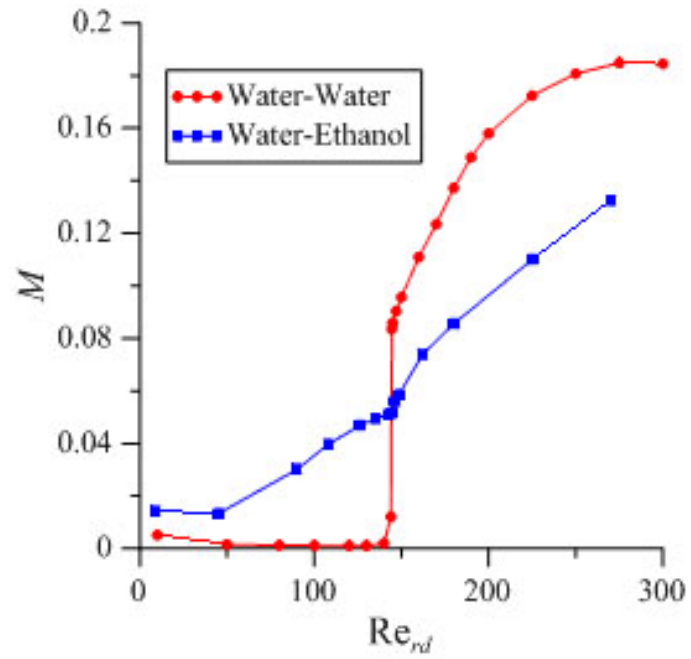

a)

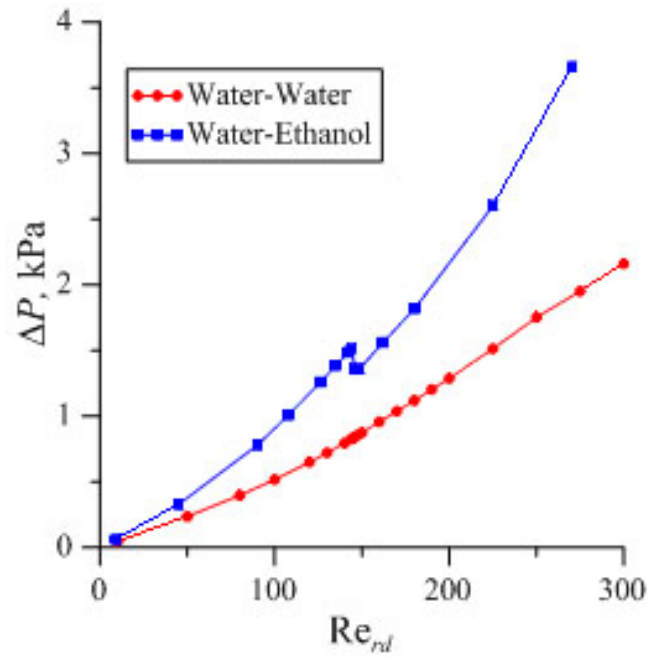

b)

Fig. 7. Water-water and water-ethanol mixing efficiency (a) and pressure drop in the mixing channel (b) dependence on reduced Reynolds number

of the thermophysical mixture properties on the concentration of ethanol is much higher than for the water-water mixing. This is due to a nonlinear increase in viscosity and a decrease in the density of the mixture with an increase in the concentration of ethyl alcohol. The presence of an inflection of the dependence of the pressure drop on the Reynolds number for the nonlinear case is explained by the similar manner. It can be seen from the graph that this inflection corresponds to transition of the flow in a micromixer to the engulfment regime. As it was shown above (see Figs. 6c-6d), an abrupt change in the flow pattern is observed at the onset of the transition, the mixing efficiency also increases abruptly, accordingly, the volume occupied by $50 \%$ mixture concentration increases, and the volume occupied by pure fluids decreases. The density and viscosity of the mixture change in the same way (jump). It leads to the behaviour of the pressure drop versus the Reynolds number for the nonlinear properties case as is shown in Fig. $7 \mathrm{~b}$.

\section{Conclusions}

The study of flow and mixing regimes of ethanol and water using numerical simulation was carried out, and the dependence of the mixing efficiency of these fluids on the Reynolds number was defined. The obtained results were compared with the case of mixing of two fluids with the same thermophysical properties. The conducted numerical simulation allowed identifying following regimes for incompressible fluid flow in T-shaped micromixer: stationary vortex-free flow; stationary symmetric vortex flow; intermittent transition from symmetric to asymmetric flow (engulfment flow); and stationary asymmetric vortex flow.

It has been shown that the differences in the densities and viscosities of the miscible fluids significantly influence on the mixing efficiency, but the listed above flow regimes with some differences can be found for water-ethanol mixing. It was found that the difference in viscosities of the miscible fluids leads to a shift of flow regimes and the difference in densities does not lead to such shift. However, a significant flow asymmetry in the region before its transition to the engulfment flow mode was observed. 
It has been also shown that the difference in viscosities leads to an asymmetry of the length of the double-vortex structure developed near the left end wall of the mixer in the stationary symmetric vortex flow regime. Besides, it has been found that each of the double-vortex structures lose symmetry with respect to the horizontal plane when approach the transition point, and at the moment of transition one of the vortices in each of the fluids begins to predominate and the second to attenuate. As a result, two weaker vortices merge into one large vortex, and the flow structure actually converts into a three-vortex system. In the engulfment regime the transitional three-vortex structure converts into a two-vortex structure. It was found that the flow and mixing of two fluids with different thermophysical properties can be considered as self-similar pattern with regard to the reduced Reynolds number obtained using the appropriate coefficient.

It has been found that the nonlinear behaviour of thermophysical properties of mixture significantly influenced on the mixing efficiency and, especially, on the pressure drop in the engulfment regime. The pressure drop in such case is much higher than for the water-water mixing. Moreover, the inflection of the dependence of the pressure drop on the Reynolds number for the water-ethanol mixing has been observed at the transition of the flow in a micromixer to the engulfment regime. This is due to a nonlinear increase in viscosity and a decrease in the density of the mixture with an increase in the concentration of ethanol.

The reported study was funded by Russian Foundation for Basic Research, Government of Krasnoyarsk Territory, Krasnoyarsk Regional Fund of Science to the research project no. 18-48243011: "The calculation study of ways to enhance the efficiency of microfluidic reactors» and by the Ministry of Education and Science of the Russian Federation Government contract with Siberian Federal University in 2018 (no. 16.8368.2017)

\section{References}

[1] P.Tabeling, Introduction to microfluidics, Oxford, Oxford University Press, 2005

[2] G.Karnidakis, A.Beskok, N.Aluru, Microflows and nanoflows, Interdisciplinary Applied Math. Springer Science+Business Media Inc., Vol. 29, 2005.

[3] R.Karnik, Microfluidic mixing Encyclopedia of microfluidics and nanofluidics, Ed. Li D., Springer, 2008.

[4] V.Rudyak, A.Minakov, Modeling and optimization of Y-type micro-mixers, Micromachines, 5(2014), no. 4, 886-912.

[5] J.Aubin, D.F.Fletcher, C.Xuereb, Design of micro-mixers using CFD modeling, Chem. Eng. Sci., 60(2005), no. 8-9, 2503-2516.

[6] M.Hoffmann, M.Schluter, N.Rubiger, Experimental investigation of fluid-fluid mixing in Tshaped micro-mixers using $\mu$-LIF and $\mu$-PIV, Chem. Eng. Sci., 61(2006), no. 9, 2968-2976.

[7] D.Bothe, C.Stemich, H.-J.Warnecke, Theoretical and experimental investigations of mixing processes in T-shaped microreactors - Part 1: Numerical simulation and assessment of flow mixing, Chemie Ingenieur Technik, 76(2004), no. 10, 1480-1484. https://doi.org/10.1002/cite.200400085 
[8] D.Bothe, C.Stemich, H.-J.Warnecke, Fluid mixing in a T-shaped micro-mixer, Chem. Eng. Sci., 61(2006), no. 9, 2950-2958.

[9] C.Stemich, Theoretische und numerische Untersuchung des Struomungsmischens in einem T-formigen Mikromischer, Dissertation zur Erlangung des akademischen Grades Doktor der Naturwissenschaften, Universitat at Paderborn, 2006.

[10] S.Dreher, N.Kockmann, P.Woias, Characterization of laminar transient flow regimes and mixing in T-shaped micro-mixers, Heat Transfer Engineering, 30(2009), no. 1-2, 91-100.

[11] A.V.Minakov, V.Ya.Rudyak, A.A.Gavrilov, A.A.Dekterev, Mixing in T-shaped micromixer at moderate Reynolds numbers, Thermophysics and Aeromechanics, 19(2012), 385-395.

[12] A.Minakov, V.Rudyak, A.Dekterev, A.Gavrilov, Investigation of slip boundary conditions in the T-shaped microchannel, Int. J. of Heat and Fluid Flow, 43( 2013), 161-169.

[13] A.Minakov, A.Yagodnitsina, A.Lobasov, V.Rudyak, A.Bilsky, Study of fluid flow in micromixer with symmetrical and asymmetrical inlet conditions, La Houille Blanche, (2013), no. $5,12-21$.

[14] A.S.Lobasov, A.V.Minakov, V.Ya.Rudyak, Viscosity effect on the flow modes in T-type micro-mixers, Fluid Dynamics, 51(2016), no. 3, 381-388.

[15] A.S.Lobasov, A.V.Minakov, Density effect on the mixing efficiency and flow modes in Tshaped micromixers, MATEC Web of Conferences, 115(2017), 07002.

[16] A.S.Lobasov, A.A.Shebeleva, Initial temperatures effect on the mixing efficiency and flow modes in T-shaped micromixer, J. Phys.: Conf. Ser., 899(2017), 022010.

[17] C.Galletti, G.Arcolini, E.Brunazzi, R.Mauri, Mixing of binary fluids with compositiondependent viscosity in a T-shaped micro-device, Chem. Eng. Sci., 123(2015), 300-310.

[18] C.Galletti, E.Brunazzi, R.Mauri, Unsteady mixing of binary liquid mixtures with composition-dependent viscosity, Chem. Eng. Sci., 164(2017), 333-343.

[19] G.Orsi, M.Roudgar, E.Brunazzi, C.Galletti, R.Mauri, Water-ethanol mixing in T-shaped microdevices, Chem. Eng. Sci., 95(2013), 174-183.

[20] W.Wang, S.Zhao, T.Shao, Y.Jin, Y.Cheng, Visualization of micro-scale mixing in miscible liquids using $\mu$-LIF technique and drug nano-particle preparation in T-shaped microchannels, Chem. Eng. J., 192(2012), 252-261.

[21] R.J.Poole, M.Alfateh, A.P.Gauntlett, Bifurcation in a T-channel junction: Effects of aspect ratio and shear-thinning, Chem. Eng. Sci., 104(2013), 839-848.

[22] T.Andreussi, C.Galletti, R.Mauri, S.Camarri, M.V.Salvetti, Flow regimes in T-shaped micro-mixers, Comp. and Chem. Eng., 76(2015), 150-159.

[23] A.Soleymani, H.Yousefi, I.Turunen, Dimensionless number for identification of flow patterns inside a T-micromixer, Chem. Eng. Sci., 63(2008), no. 21, 5291-5297.

[24] A.Fani, S.Camarri, M.V.Salvetti, Investigation of the steady engulfment regime in a threedimensional T-mixer, Phys. Fluids, 25(2013), no. 6, 064102 
[25] A.Fani, S.Camarri, M.V.Salvetti, Unsteady asymmetric engulfment regime in a T-mixer, Physics of Fluids, 26(2014), no. 7, 074101.

[26] C.Galletti, M.Roudgar, E.Brunazzi, R.Mauri, Effect of inlet conditions on the engulfment mode in a T-shaped micro-mixer, Chem. Eng. J., 185(2012), 300-313.

[27] A.A.Gavrilov, A.V.Minakov, A.A.Dekterev, V.Ya.Rudyak, A numerical algorithm for modeling laminar flows in an annular channel with eccentricity, J. of App. and Ind. Math., 5(2011),no. 4, 559-568.

[28] S.Patankar, Numerical methods in heat transfer and fluid dynamics, Moscow, Energoatomizdat, 1984 (in Russian).

[29] Yu.A.Bystrov, S.A.Isaev, K N.A.udryavtsev, A.I.Leont'ev, Numerical simulation of vortex based enhancement of heat transfer in tube packages, Shipbuilding, 2005.

[30] J.H.Ferziger, M.Peric, Computational methods for fluid dynamics, Berlin, Springer Science+Business Media Inc., 2002.

[31] B.P.Leonard, A stable and accurate convective modeling procedure based on quadratic upstream interpolation, Comp. Math. Appl. Mech. Eng., 19(1979), no. 1, 59-98.

[32] C.M.Rhie, W.L.Chow, Numerical study of the turbulent flow past airfoil with trailing edge separation, AIAA Journal, 21(1983), no. 11, 1525-1532.

[33] U.Trottenberg, C.W.Oosterlee, A.Schbller, Multigrid, Academic Press, 2001.

[34] M.Dizechi, E.Marschall, Viscosity of some binary and ternary liquid mixtures, J. Chem. Eng. Data, 27(1982), 358363

\title{
Изучение режимов смешения воды и этанола в Т-образных микромиксерах
}

\author{
Александр С. Лобасов \\ Анна А. Шебелева \\ Андрей В. Минаков \\ Институт инженерной физики и радиоэлектроники \\ Сибирский федеральный университет \\ Свободный, 79, Красноярск, 660041 \\ Россия
}

В работе с помощъю численного моделирования проведено изучение режимов смешения этанола и воды и определена зависимость эффективности смешения этих жидкостей от числа Рейнолъдса. В ходе численного исследования было обнаружено существенное влияние различий в вязкостях и плотностях смешивающихся жидкостей на режимы течения и эффективность смешения этих двух жидкостей. Получены распределения давления, скоростей и концентрации компонент в микромиксере. Определена зависимость эффективности смешения жидкостей и перепада давлений от числа Рейнольдса, а также построена карта режимов течения и смешения. Учитываласъ нелинейная зависимость теплофизических свойств смеси от концентрации этанола. Было обнаружено, что в таком случае наблюдается существенно большее значение перепада давлений по сравнению со случаем смешения вода-вода. Кроме того, наблюдался перегиб на грабике зависимости перепада давлений от числа Рейнолъдса для смешения вода-этанол при переходе ко втягивающему режиму течения в микромиксере.

Ключевые слова: гидродинамика, численное моделирование, Т-образный микромиксер, микромиксер, этанол, нелинейность. 